\title{
Optimized multilayer dielectric mirror coatings for gravitational wave interferometers
}

Juri Agresti, Giuseppe Castaldi, Riccardo DeSalvo, Vincenzo Galdi, Vincenzo Pierro, et al.

Juri Agresti, Giuseppe Castaldi, Riccardo DeSalvo, Vincenzo Galdi, Vincenzo Pierro, Innocenzo M. Pinto, "Optimized multilayer dielectric mirror coatings for gravitational wave interferometers," Proc. SPIE 6286, Advances in Thin-Film Coatings for Optical Applications III, 628608 (28 August 2006); doi: $10.1117 / 12.678977$

SPIE. Event: SPIE Optics + Photonics, 2006, San Diego, California, United States 


\title{
Optimized multilayer dielectric mirror coatings for gravitational wave interferometers
}

\author{
Juri Agresti ${ }^{a, b}$, Giuseppe Castaldi ${ }^{c}$, Riccardo DeSalvo ${ }^{a}$, Vincenzo Galdi ${ }^{c}$, \\ Vincenzo Pierro ${ }^{c}$, and Innocenzo M. Pinto ${ }^{c}$ \\ ${ }^{a}$ LIGO Laboratory, California Institute of Technology, Pasadena, CA 91125, USA; \\ ${ }^{b}$ Department of Physics, University of Pisa, I-56127, Pisa, Italy; \\ ${ }^{c}$ Waves Group, Department of Engineering, University of Sannio, I-82100, Benevento, Italy
}

\begin{abstract}
The limit sensitivity of interferometric gravitational wave antennas is set by the thermal noise in the dielectric mirror coatings. These are currently made of alternating quarter-wavelength high/low index material layers with low mechanical losses. The quarter-wavelength design yields the maximum reflectivity for a fixed number of layers, but not the lowest noise for a prescribed reflectivity. This motivated our recent investigation of optimal thickness configurations, which guarantee the lowest thermal noise for a targeted reflectivity. This communication provides a compact overview of our results, involving nonperiodic genetically-engineered and truncated periodically-layered configurations. Possible implications for the advanced Laser Interferometer Gravitational wave Observatory (LIGO) are discussed.
\end{abstract}

Keywords: Multilayer coatings, dielectric mirrors, gravitational waves, interferometers, thermal noise.

\section{INTRODUCTION}

Interferometric gravitational wave (GW) detectors like LIGO, ${ }^{1}$ VIRGO, ${ }^{2}$ GEO,${ }^{3}$ and TAMA $^{4}$ are very-long-baseline optical interferometers featuring multilayer dielectric mirrors. These consist of a suitable number of alternating layers of high- and low-refractive-index materials, successively grown by ion-sputtering starting off a substrate (the mirror body). ${ }^{*} \mathrm{Bulk}$ (fused) $\mathrm{SiO}_{2}$ (silica) is presently in use for the mirror body, in view of its very low thermal noise (TN); ionsputtered $\mathrm{SiO}_{2} / \mathrm{Ta}_{2} \mathrm{O}_{5}$ (tantala) are used for the low- and high-index coating layers, in view of their mild mechanical and optical losses and good dielectric contrast. ${ }^{5}$

The mirror reflectivity determines the cavity finesse (i.e., loosely speaking, the effective interferometer arm-length) and should be accordingly kept as high as possible. On the other hand, the overall limit sensitivity of the instrument is bounded by the noise floor, ${ }^{* *}$ which, in the most interesting observational frequency band (between $\sim 30$ and $\sim 300 \mathrm{~Hz}$ ), is dominated by the Brownian $\mathrm{TN}$ of the mirror coating ${ }^{+}$(internal friction in the coating layers), in all presently operating (and possibly future) interferometers. ${ }^{7,8}$

All interferometers presently in operation use quarter-wavelength (QWL) coating designs, which are known (Bragg theorem) to be optimal, in the sense that they yield the largest reflectivity for any fixed number of layers (or, equivalently, the smallest number of layers for any prescribed reflectivity). However, QWL coatings do not yield the minimum TN for a prescribed reflectivity, and hence are not optimal for GW interferometers, where the quantity that should be maximized is the visibility distance of the instrument.

This paper addresses the above optimization problem, and is organized as follows. In Section 2, we outline our working model for the multilayer mirror reflectivity and TN. In Section 3, we present several examples of optimized coatings, starting from nonperiodic genetically-engineered configurations and subsequently focusing on periodic stacked-doublet multilayers (with possible tweaking of the terminal layers), and compare them with the standard QWL syntheses. Conclusions and recommendations follow in Section 4.

\footnotetext{
* A terminating half-wavelength low-index layer (cap) is also added, for protective purposes.

** A numerical code for computing the LIGO noise curves is available at http://www.ligo.mit.edu/bench/bench.html

${ }^{+}$An introductory discussion of the various TN components in the mirrors can be found in Ref. 6 .
}

Advances in Thin-Film Coatings for Optical Applications III, edited by Michael J. Ellison, Proc. of SPIE Vol. 6286, 628608, (2006) · 0277-786X/06/\$15 - doi: 10.1117/12.678977 


\section{BACKGROUND}

\subsection{Multilayer Coating Reflectivity}

We consider a planar multilayer dielectric coating composed of stacked homogeneous layers illuminated by a normallyincident plane wave, with implied time-harmonic dependence $\exp (\iota \omega t)$. The transmission matrix for the $m$-th dielectric layer, relating the (complex) electric and magnetic field amplitudes $E$ and $H$ at its terminal planes, labeled by suffixes $m$ and $m+1$, can be written as ${ }^{9}$

$$
\left[\begin{array}{l}
E_{m} \\
Z_{0} H_{m}
\end{array}\right]=\left[\begin{array}{ll}
\cos \delta_{m} & \imath n_{m}^{-1} \sin \delta_{m} \\
l n_{m} \sin \delta_{m} & \cos \delta_{m}
\end{array}\right] \cdot\left[\begin{array}{l}
E_{m+1} \\
Z_{0} H_{m+1}
\end{array}\right], m=1,2, \ldots, N,
$$

where $\delta_{m}$ and $n_{m}$ denote the phase thickness and refraction index of the layer, respectively, and $Z_{0}=\sqrt{\mu_{0} / \varepsilon_{0}}$ is the vacuum characteristic impedance. The input reflection coefficient of the multilayer is obtained by chain-multiplying the transmission matrices of its layers, starting from the substrate surface (where $E_{N}=n_{S}^{-1} Z_{0} H_{N}, n_{S}$ being the substrate refractive index), so as to obtain the input impedance $Z_{i}$ of the coating at mirror-vacuum interface $(m=l)$. The reflection coefficient is then simply ${ }^{9}$

$$
\Gamma=\frac{Z_{0}-Z_{i}}{Z_{0}+Z_{i}}
$$

The above formalism can be easily generalized to the case of oblique incidence, via the introduction of (polarizationdependent) "transverse" wave-impedances and refractive indexes ${ }^{9}$.

For (truncated) periodic coatings made of cascaded identical cells, the transmission matrix describing the unit cell,

$$
\left[\begin{array}{l}
E_{m} \\
Z_{0} H_{m}
\end{array}\right]=\left[\begin{array}{ll}
A & B \\
C & D
\end{array}\right] \cdot\left[\begin{array}{l}
E_{m+1} \\
Z_{0} H_{m+1}
\end{array}\right],
$$

can likewise be written as the (ordered) product of the transmission matrices representing its component layers. For these configurations (see also Section 3.2 below), a major role is played by the eigenvalues of the above transmission matrix, which are most conveniently written as exponentials, i.e.,

$$
\frac{E_{m+1}}{E_{m}}=\frac{H_{m+1}}{H_{m}}=\exp \xi
$$

where $\xi$ is the Bloch characteristic exponent (BCE), ${ }^{10}$ which is obtained by solving

$$
\cosh \xi=\frac{A+D}{2}
$$

In the absence of optical losses, this equation admits two (either real or purely imaginary) solutions differing only by sign. A lossless infinite periodic structure exhibits an infinite sequence of alternating frequency bands where propagation is either allowed (transmission-bands) or forbidden (reflection-bands, or band-gaps), according to whether its BCE is either purely imaginary or purely real. The larger $|\operatorname{Re}(\xi)|$, the better will a (lossless) truncated-periodic coating made of cascaded identical cells behave as a mirror.

\subsection{Coating Thermal Noise}

The coating TN power spectral density (PSD) may be well approximated ${ }^{11}$ by a weighted sum over the thicknesses of the two materials: 


$$
\bar{v}=C\left(S_{T}+\gamma^{-1} S_{S}\right)
$$

where $S_{T}, S_{S}$ are the total (physical) thicknesses of the high/low index coating materials, respectively, $C$ is a (dimensional) constant, and $\gamma$ is the ratio between their (specific) mechanical noises. Equation (6) can be obtained starting from the rigorous coating TN PSD representation obtained in Refs. 12,13. The specific noise ratio $\gamma$ in (6) is of the order of $Y_{T} \phi_{T} / Y_{S} \phi_{S}, \quad Y_{T, S}$ and $\phi_{T, S}$ being the Young moduli and mechanical loss-angles, respectively, of the tantala and silica.

Loss angle measurements are unfortunately affected by relatively large uncertainties, related both to the measurement procedure (perhaps Poissonian-distributed) and to technological repeatability (perhaps Gaussian-distributed). Uncertainties also exist for the coating $Y_{T, S}$ values, which are presently approximated by the corresponding bulk material values. A sensible systematic difference between the silica loss angle values in Ref. 13 as compared to Ref. 12 is also noted, mainly as an effect of more accurate numerical modeling. The value of $\gamma$ to be used in (6) is thus rather uncertain. The results presented in this paper are based on the representative conservative assumption $\gamma=10$, for plain silica/tantala coatings.

\section{OPTIMIZED COATINGS}

\subsection{Genetically-Engineered Configurations}

Genetic algorithms (GAs) are credited as among the most robust and reliable no-derivative global-optimization tools available, for problems of moderate size (up to a few tens unknowns). ${ }^{14}$ In particular, GAs allow:

- To accommodate multi-objective and/or multiple constraint prescriptions, even non-differentiable ones, combining continuous (e.g., the layers thicknesses) as well as discrete (e.g., the number of layers) optimization parameters;

- To adopt a controlled ignorance attitude (using a minimum of a priori assumptions on the form of the sought solutions), and to obtain hints about this latter upon inspection of the outcomes.

GAs have proven as effective tools for synthesizing general (e.g., multi-dielectric) reflective coatings, featuring several heterogeneous (e.g., technological) design constraints, with multiobjective (e.g., multiple wavelength) optimization targets. ${ }^{15}$ We implemented a GA engine based coating optimization (FORTRAN) code. The PIKAIA engine by P. Charbonneau ${ }^{16}$ was chosen in view of its free availability, as well as its updated, flexible and well-documented architecture. ${ }^{17}$ We performed a series of numerical experiments using the above GA code, first for the simplest case where the (absolute) reflection coefficient is maximized for a fixed number of layers, and a prescribed upper bound for the total amount of tantala in the coating (see, e.g., Fig. 1 and Table I), and then for the more realistic case where the silica losses are taken into account and the overall noise is constrained instead. The following common features were observed in the genetically evolved "solutions":

- As the number of generations is increased, the centermost part of the GA-optimized coatings exhibits a neat tendency to become a cascade of almost identical doublets, whose (total) optical lengths cluster around $\lambda / 2$;

- Sensible deviations from the above are mostly confined among the terminal coating layers;

- The coating reflection coefficient is maximum when the whole allowed amount of tantala is used;

- The number of generations needed for GA to "converge" is a steeply increasing functions of the problem size.

The above findings led us to investigate the performance of coatings composed of cascaded identical multiplets of relatively small size. 
$\mathrm{Ta}_{2} \mathrm{O}_{5}$ layer \#

$\begin{array}{llllllllllllllllllllll}2 & 4 & 6 & 8 & 10 & 12 & 14 & 16 & 18 & 20 & 22 & 24 & 26 & 28 & 30 & 32 & 34 & 36 & 38 & 40 & 42 & 44\end{array}$

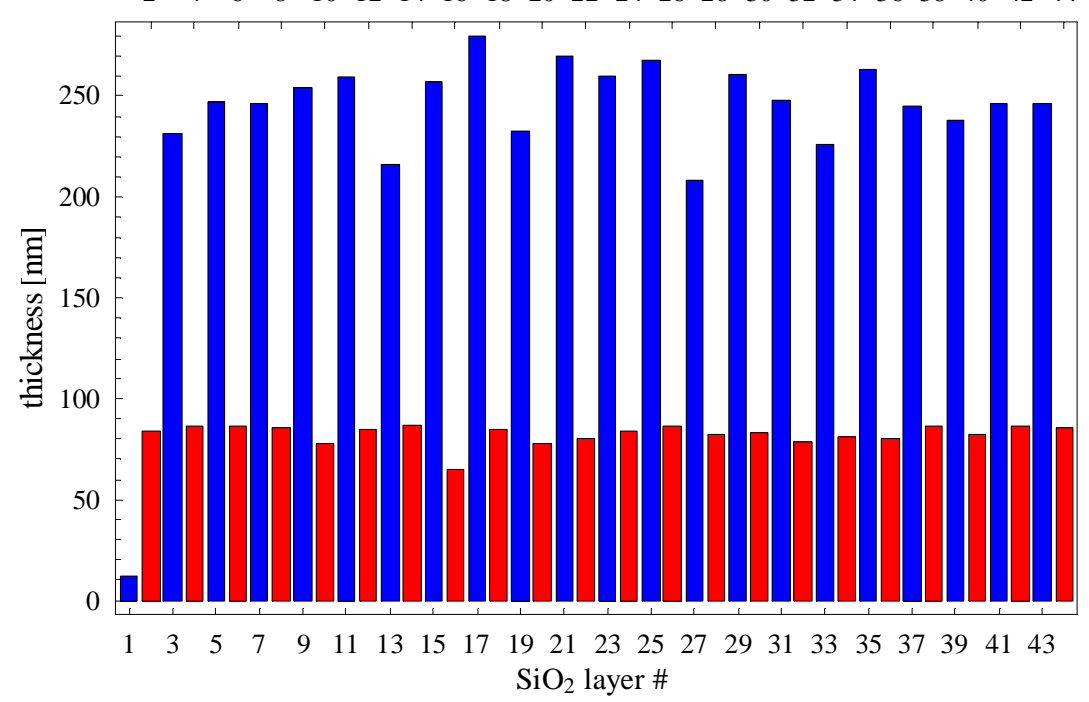

Figure 1. Genetically engineered mirror prototype (best design after $10^{5} \mathrm{GA}$ generations). Design goals: Transmissivity $1-|\Gamma|^{2} \leq 15$ ppm; tantala thickness $\leq 80 \%$ of the amount used in standard QWL design with closest reflectivity.

\begin{tabular}{|c|c|c|c|}
\cline { 2 - 4 } \multicolumn{1}{c|}{} & QWL1 & GA & QWL2 \\
\hline $\begin{array}{c}\text { \# of layers } \\
\text { (cap included) }\end{array}$ & 36 & 44 & 28 \\
\hline $1-|\Gamma|^{2}[\mathrm{ppm}]$ & 16.20 & 14.91 & 235.46 \\
\hline$S_{T}[\mathrm{~nm}]$ & 2359.43 & 1815.61 & 1835.11 \\
\hline$S_{S}[\mathrm{~nm}]$ & 5839.41 & 7033.01 & 4582.46 \\
\hline Relative PSD & 1.17 & 1 & 0.91 \\
\hline
\end{tabular}

Table I. Comparison between GA-optimized prototype in Fig. 1 and nearest QWL designs in terms of reflectivity (QWL1) or tantala content (QWL2).

\subsection{Stacked Doublet Coatings}

Stacked doublet coatings are the most natural generalization of the standard QWL designs. For optically lossless cascaded-multiplet coatings one can prove that the (absolute) coating reflection coefficient converges to unity as the total number of doublets increases without bound, irrespective of the refraction indexes of the terminating media (mirror substrate, vacuum), in the stop-bands of the single multiplet transmission matrix. The rate of this convergence is ruled by the BCE in (6) (the larger the BCE, the faster the convergence). Remarkably, one can verify that if only two different refractive media are used, it is impossible to design a $2 M$-layer multiplet featuring either a larger $\mathrm{BCE}$ value with the same TN, or a smaller TN with the same BCE value, as compared to all multiplets consisting of $M$ equal cascaded doublets. Thus, no cascade of $K$ equal $2 M$-layer multiplets can outperform (in the above sense) some cascade of $K \times M$ 
equal doublets. The study of truncated periodically-layered mirrors can thus be restricted to the simplest case of (truncated) equal cascaded doublets.

The BCE and the TN of a multilayer coating composed of $N$ identical cascaded doublets are functions of the doublet optical (i.e., scaled to the local wavelength) thicknesses $z_{S}$ and $z_{T}$ only. This results in the following simple algorithm for drawing the general trade-off design curves displayed in Figs. 2 and 3:

- Assign the number $N$ of doublets;

- Assign the upper-bound TN of the whole coating;

- Compute the corresponding upper-bound doublet TN;

- Determine $z_{S}$ and $z_{T}$ so as to maximize the doublet BCE under the above TN constraint.

The last step in the algorithm, once $z_{S}$ and $z_{T}$ have been determined, consists of computing the overall multilayer reflectivity. A number of representative regions corresponding to possible doublet $\mathrm{TN}$ constraints (shaded areas), and a few constant BCE contours (squeezed ellipse-like curves) in the $z_{S}-z_{T}$ plane are shown in Fig. 4.

The last step in the algorithm corresponds to finding the point on the (straight) borderline of the allowed TN region where the BCE is maximum, i.e., the BCE contour tangent to the TN borderline, as illustrated in Fig. 5(a). This also corresponds to the point on that particular BCE contour featuring the minimum doublet TN, as can be seen from Fig. 5(a). One can also observe from Fig. 5(b) that the sought points are very close to the $z_{S}+z_{T}=\lambda / 2$ line, the BCE contour lines being rather close to that line. Indeed, for all practical purposes one can assume (as actually done in drawing Figs. 2 and 3) $z_{S}+z_{T}=\lambda / 2$, which is the well-known Bragg condition.

The trade off curves in Figs. 2 and 3 contain all the information needed to optimize the design of a cascaded-doublet multilayer. Here, "optimal" means that no other multilayer composed of $N$ equal cascaded doublet featuring a prescribed coating TN will exhibit a larger (absolute) reflection coefficient, when inserted between the same terminal media.

By inspection of the curves in Fig. 3, it can be understood that for any fixed value of the mirror reflectivity there exists an optimum value of $N$ (and of the ratio $z_{T} / z_{S}$ ) yielding the minimum TN. Indeed, beyond a certain $N$ (below a certain value of the ratio $z_{T} / z_{S}$ of the order of $\gamma^{-1}$ ) the TN will eventually start growing. This is exemplified in Fig. 6, for the special case $1-|\Gamma|^{2}=8.3 \mathrm{ppm}$. The bullets and circles in Fig. 6 refer to the exact doublet optimization algorithm and approximate (thickness ratio optimized, doublet thickness $=\lambda / 2$ ) one, respectively. It is observed that the already mentioned uncertainty (assumed to be $\pm 3 \%$ in Fig. 6) in the value of the specific noise ratio $\gamma$ blurs the distinction between the full and approximate procedure.

\subsection{Tweaked Cascaded-Doublet Configurations}

The coating reflection coefficient can still be improved by tweaking the thickness of a few terminal layers, at the expense of a comparatively almost irrelevant raise in the coating TN. To explore this possibility, we used again our GA engine. Figure 7 illustrates the effect of tweaking the first (silica) and last (tantala) layer, for the special case of the minimum-noise 23-doublet $8.3 \mathrm{ppm}$ mirror (the minimum noise point in Fig 6). In this case, the transmissivity can be further reduced by some $10 \%$, while increasing the $\mathrm{TN}$ by $\sim 1 \%$. Tweaking the next to first (respectively, last) terminal layer produces less dramatic improvements. Going beyond this stage does not seem to provide worthwhile improvements.

\footnotetext{
$\dagger$ In the progress of this work we became aware ${ }^{18}$ of a CSIRO proposal for using cascaded tantala/silica doublets with optical thicknesses of $(\lambda / 8,3 \lambda / 8)$ in place of the standard QWL.
} 


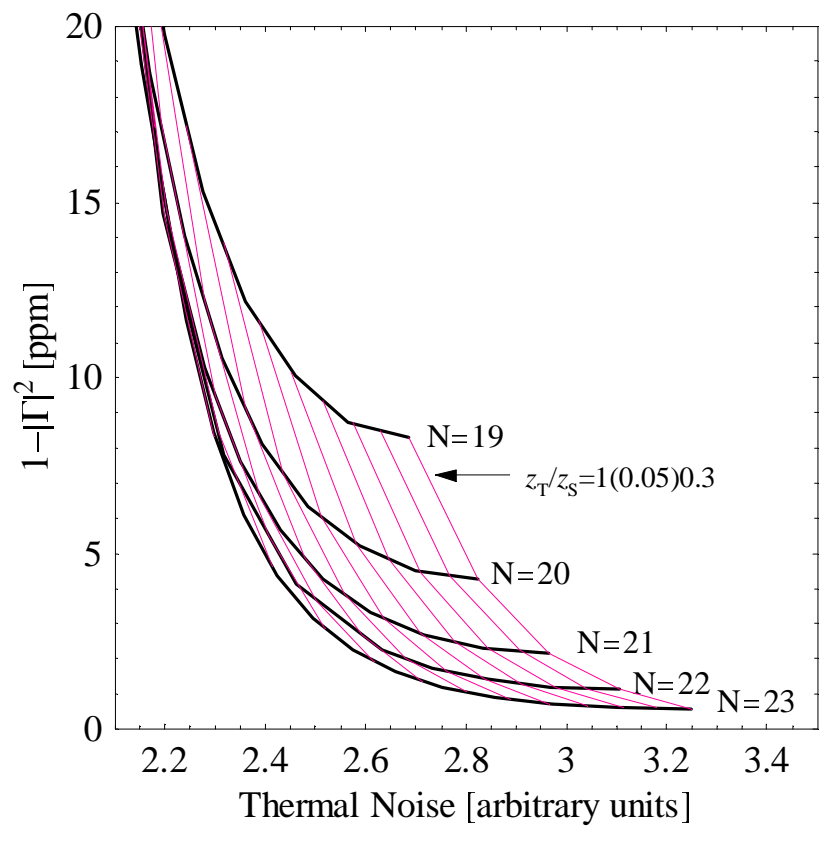

Figure 2. Cascaded-doublet coatings design nomogram. Trade-off design curves for fixed number $N$ of doublets and $\gamma$ $=10$. Points on the curves corresponding to the same value of the tantala/silica (optical) thickness-ratio are joined by thin lines. The rightmost point on each curve corresponds to the standard QWL $\left(z_{T}=z_{S}=\lambda / 4\right)$ design.

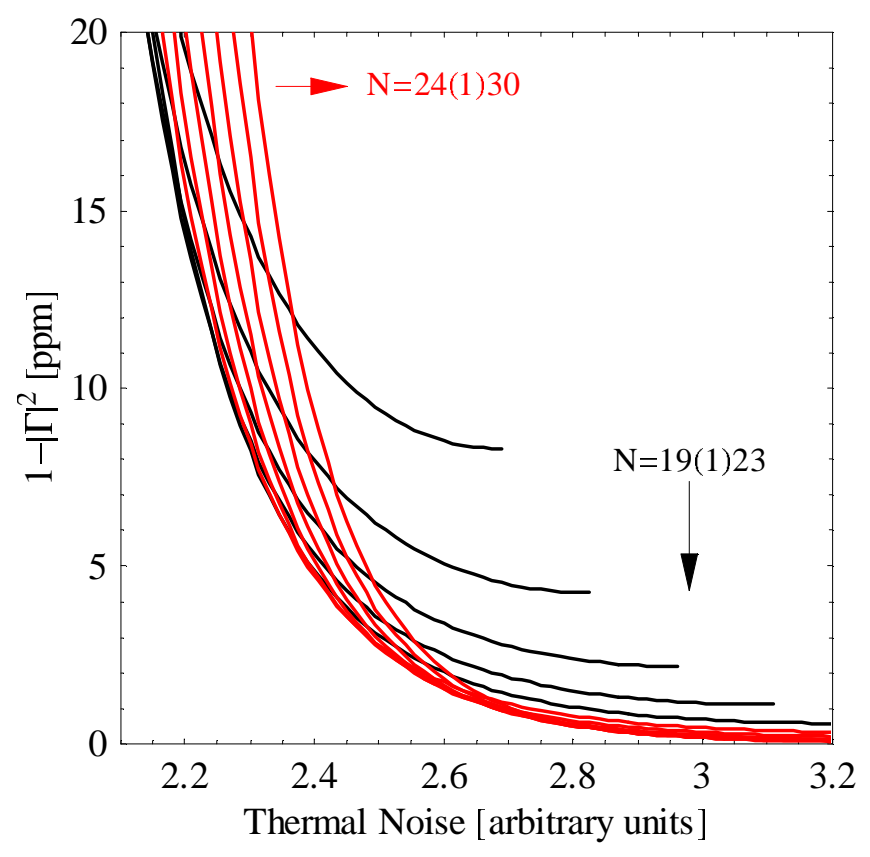

Figure 3. Cascaded doublet coatings. Trade-off design curves for several values of the number $N$ of doublets and $\gamma=10$. The rightmost point on each curve corresponds to the standard QWL design. 


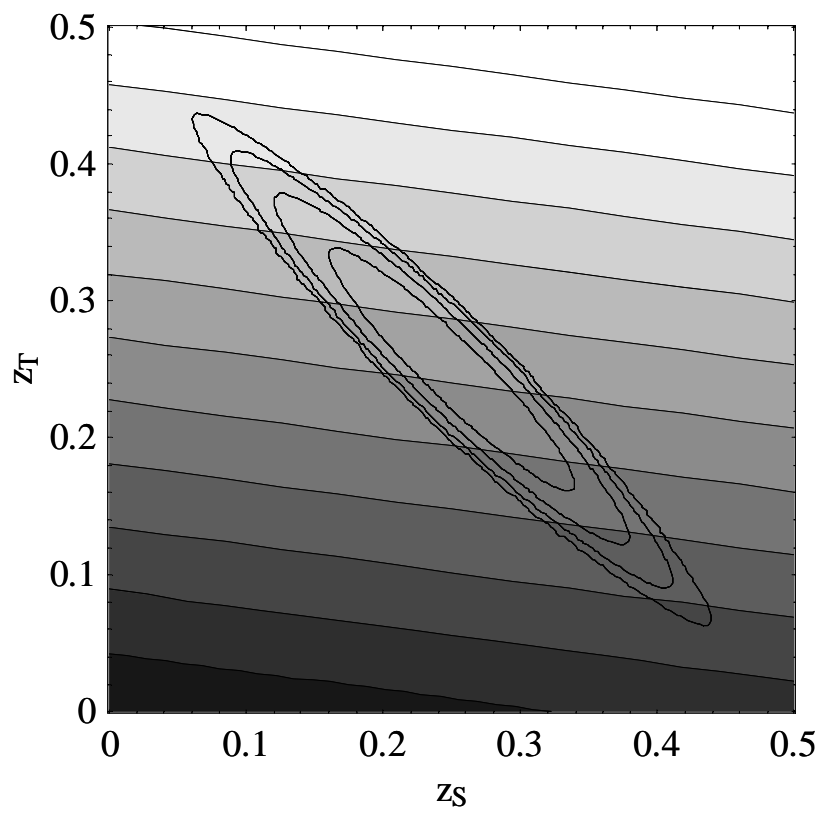

Figure 4. Cascaded doublet coating. Constant BCE contours and constant TN lines of single doublet vs. optical thickness (i.e., in units of local wavelength) of doublet layers.
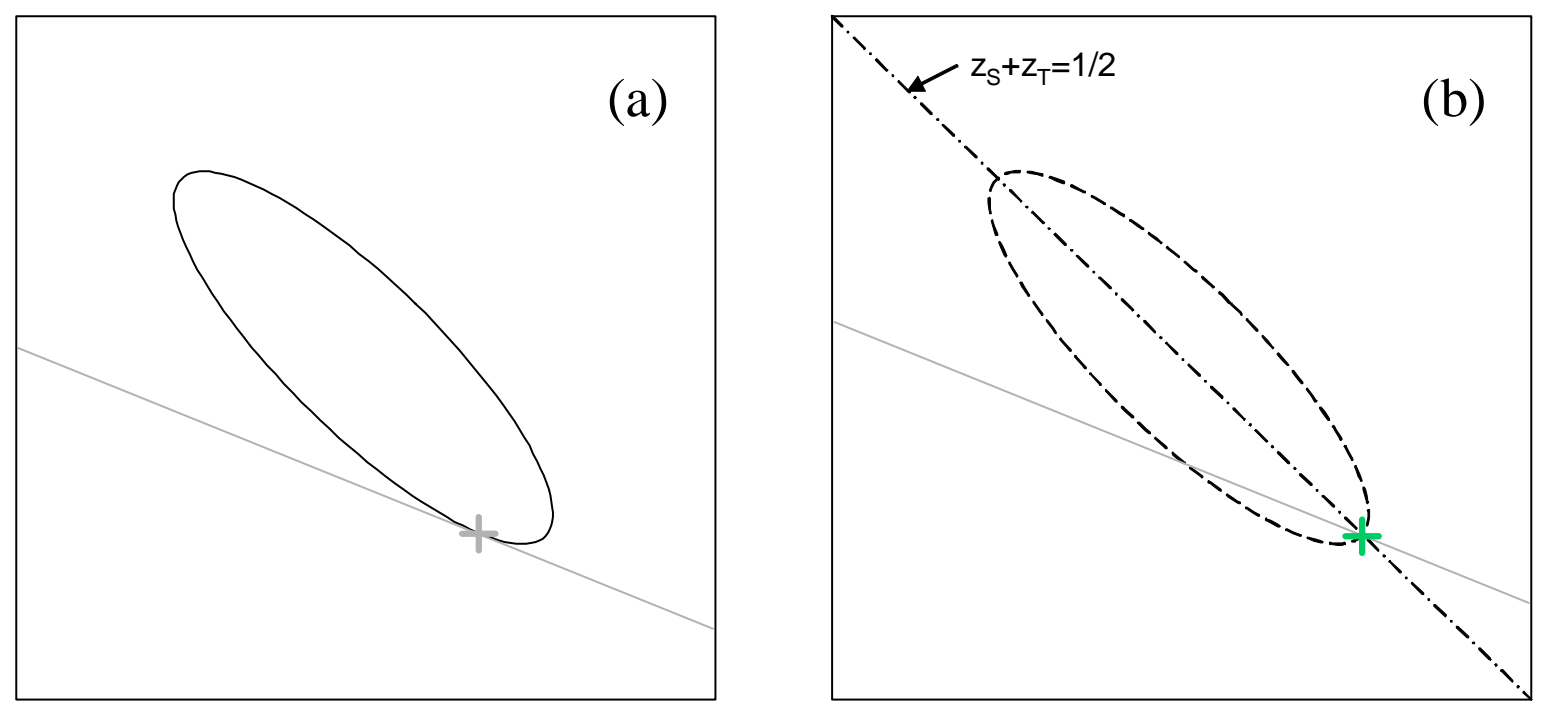

Figure 5. Cascaded doublet coating thickness optimization. Constant BCE (dark) and noise (light) contours of single doublet shown. Cross markers identify optimal designs. Equivalence between reflectivity maximization under a TN constraint, and TN minimization under a reflectivity constraint is evident. Left panel (a): Exact optimization. Right panel (b): Approximate optimization (half-wavelength doublets, thickness ratio optimized). 


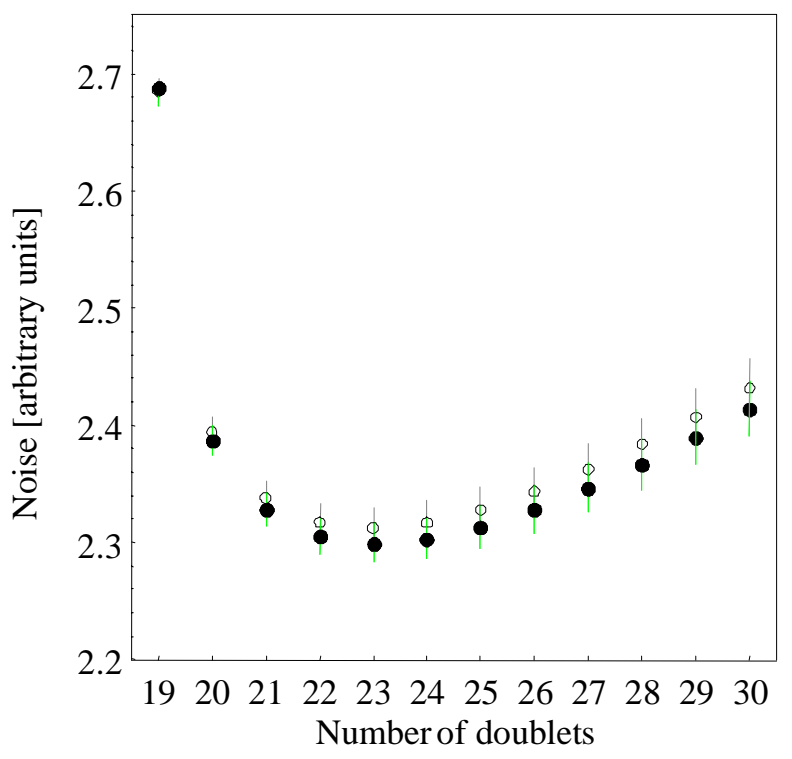

Figure 6. Cascaded doublet coating noise vs. number of doublets at $1-|\Gamma|^{2}=8.3 \mathrm{ppm}$. Circles: Half-wavelength doublets, thickness ratio optimized. Bullets: Exact optimization. Error bars correspond to $\pm 3 \%$ uncertainty in the nominal specific noise ratio $\gamma=10$.

\section{CONCLUSIONS AND RECOMMENDATIONS}

In this paper, the optimization of multilayer mirror coating for lowest noise at a prescribed reflectivity has been addressed. Brute force optimization based on evolutionary (genetic) algorithms suggested considering cascaded doublet designs. Tradeoff design curves in the form of reflectivity vs. TN curves have been obtained, whereby the optimum (optical) thicknesses of the doublet layers can be obtained.

The main result obtained by applying the above design hints is a reduction of the coating TN of the order of $14 \%$ (assuming the specific noise ratio $\gamma=10$ ) as compared to the standard QWL design, at transmissivity levels of the order of $10 \mathrm{ppm}$. Under the simplest assumptions where the GW sources are distributed homogeneously/isotropically throughout space, and their spectral power is essentially contained in the coating-noise dominated sensitivity band of the instrument, this would boost the event rate by some $25 \%$. Similar figures ${ }^{19}$ are obtained for the 1ppm AdvLIGO baseline design. ${ }^{20,21}$

The TN reduction is obtained at the expense of an affordable increase in the number of layers, entailing a reduction (increase) in the total amount of tantala (silica) used. The related power absorption, frequency and polarization response, as well as the effects of random errors in the dielectric layer thicknesses and in the measured silica/tantala mechanical loss angles were also investigated. ${ }^{19}$ These results, in parallel with the development of novel materials (titanium-doped tantala) featuring lower mechanical and optical losses, are currently being used in the fabrication of prototypes (in collaboration with the CNRS Laboratoire des Matériaux Avancés, Lyon, France). Direct TN measurements are planned with the Thermal Noise Interferometer (TNI) at the California Institute of Technology, Pasadena, CA, USA, as a collaboration with the LIGO Lab. 


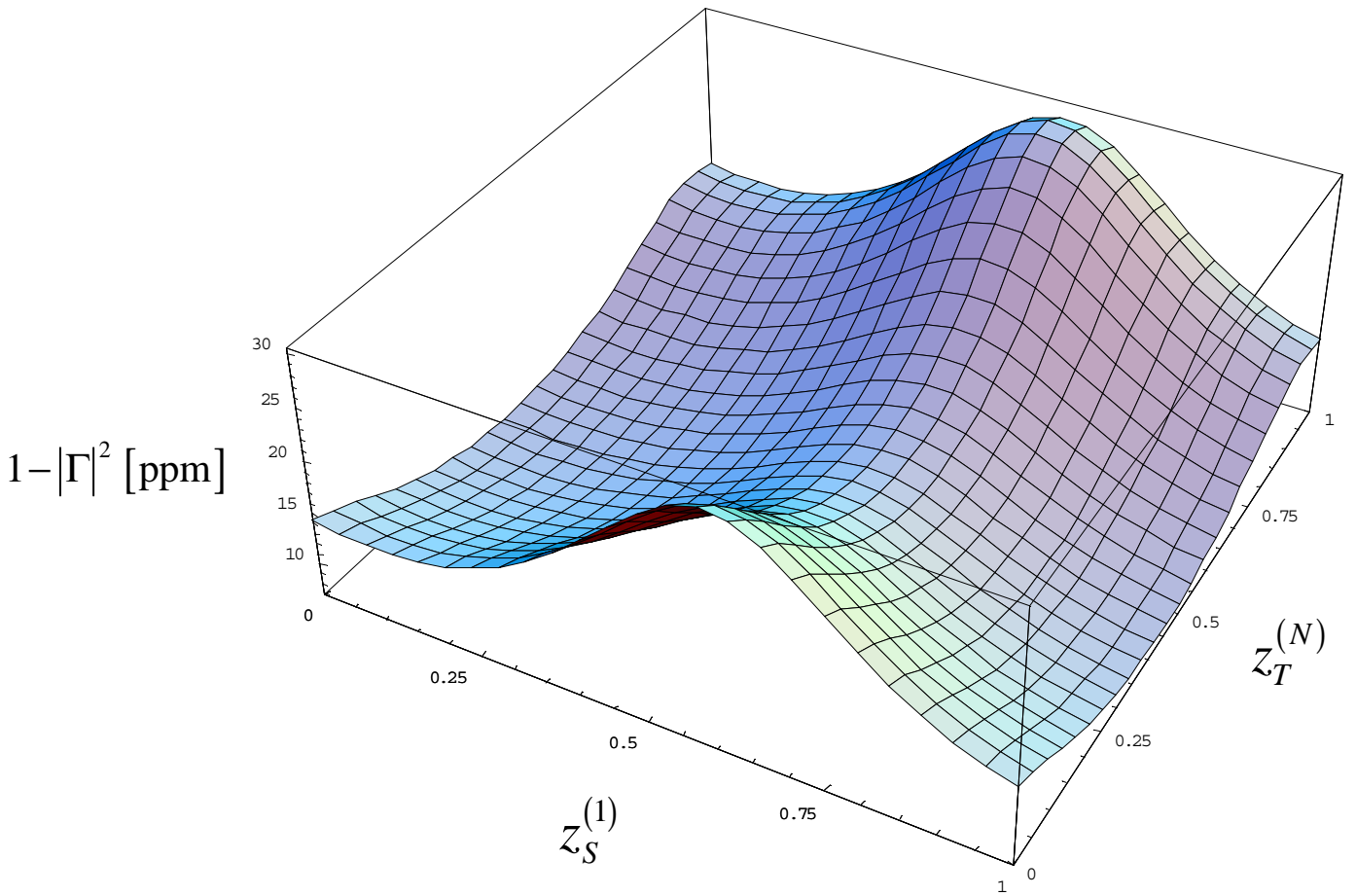

Figure 7. Cascaded 23-doublet coating. Transmissivity vs. terminal layer thickness (here, in units of $\lambda / 2$ ). Optimum values: $z_{s}^{(1)}=50.19 \mathrm{~nm}, z_{T}^{(N)}=232.56 \mathrm{~nm}$ (to be compared with $z_{s}^{(1)}=362.358 \mathrm{~nm}, z_{T}^{(N)}=164.255 \mathrm{~nm}$ for the internal layers). $1-|\Gamma|^{2}$ is accordingly reduced from 8.3 to $7.29 \mathrm{ppm}(12 \%)$. TN is increased from 2.3 to $2.33(+1.3 \%)$.

\section{ACKNOWLEDGMENTS}

This work has been performed within the frame of a MOU with the LIGO Scientific Collaboration (LSC), and has been sponsored in part by the Italian National Institute for Nuclear Physics (INFN, Gruppo V) through the COAT project. The work of J.A. and R.DS. was supported by the National Science Foundation under Grant No. PHY-0107417.

1. http://www.ligo.caltech.edu

\section{REFERENCES}

2. http://www.virgo.infn.it

3. http://www.geo600.uni-hannover.de

4. http://tamago.mtk.nao.ac.jp

5. G. M. Harry et al., "Optical coatings for gravitational wave detectors", in Advances in Thin Film Coatings for Optical Applications, J. D. T. Kruschwitz and J. B. Oliver, Eds., Proc. SPIE 5527, SPIE, Bellingham, WA, USA, 2004, pp. 33-40.

6. S. Rao, Mirror Thermal Noise in Interferometric GW Detectors, Ph.D. Thesis, California Institute of Technology, CA, USA, 2003, http://etd.caltech.edu/etd/available/etd-05092003-153759/unrestricted/thesis.pdf

7. G. M. Harry et al., "Thermal noise in interferometric gravitational wave detectors due to dielectric optical coatings," Class. Quantum Grav. 19, pp. 897-917, 2002.

8. G. M. Harry et al., "Thermal noise from optical coatings in gravitational wave detectors," Appl. Optics 45, pp. 1569-1580, 2006. 
9. S. J. Orfanidis, Electromagnetic Waves and Antennas (web-book, http://www.ece.rutgers.edu/ orfanidi/ewa/).

10. R. E. Collin, Field Theory of Guided Wave ( $2^{\text {nd }}$ edition), IEEE Press, 1990.

11. J. Agresti et al., "Optimized coatings," LIGO Scientific Collaboration (LSC) Meeting, Hanford, WA, USA, Aug. 14-17, 2005 (LIGO-G050363-00-R).

12. S. D. Penn et al., "Mechanical loss in tantala/silica dielectric mirror coatings," Class. Quantum Grav. 20, pp. $2917-$ 2928, 2003.

13. D. R. M. Crooks et al., "Experimental measurements of mechanical dissipation associated with dielectric coatings formed using $\mathrm{SiO}_{2}, \mathrm{Ta}_{2} \mathrm{O}_{5}$ and $\mathrm{Al}_{2} \mathrm{O}_{3}$," Class. Quantum Grav. 23, 2006 (in print).

14. Y. Rahmat-Samii and E. Michiessen (Eds.), Electromagnetic Optimization by Genetic Algorithms, J. Wiley \& Sons, 1999.

15. S. Lunt and R. S. Turley, "The use of genetic algorithms in multilayer mirror optimization," J. Utah Acad. 76, pp. 61-63, 1999.

16. P. Charbonneau, "Genetic algorithms in astronomy and astrophysics," Astrophys. J. (Suppl.) 101, pp. 309-317, 1995.

17. http://www.hao.ucar.edu/Public/models/pikaia/pikaia.html

18. H. Armandula, private communication, 2005.

19. J. Agresti et al., "Optimized coating status," LIGO Scientific Collaboration (LSC) Meeting, Hanford, WA, USA, Mar. 19-22, 2006 (LIGO-G060088-00-Z).

20. P. Fritschel, "Second generation instrument for the Laser Interferometer Gravitational Observatory," in Gravitational Wave Detection, M. Cruise and P. Saulson, Eds., Proc. SPIE 4856, SPIE, Bellingham, WA, USA, 2003, pp. 282-291.

21. D. Shoemaker, "Advanced LIGO: Context and overview (Proposal to the NSF)," Report LIGO-M030023-00, 2003. 